\title{
A Crystal Flask Composed of Huge Cage-of-Cage Metallosupramolecules for the Formation of Polyoxomolybdate
}

\author{
T. Kojima, B. Wahyudianto, T. Konno \\ Department of Chemistry, Graduate School of Science, Osaka University, 1-1, Machikaneyama, Toyonaka, Osaka, 560-0043, Japan \\ kojimat15@chem.sci.osaka-u.ac.jp
}

Over the last decade, crystal flask that converts one chemical species to another one in single-crystal-to-single-crystal (SCSC) manner has attracted much attention in crystal engineering. An important key for the construction of crystal flask is a porous space which can induce a chemical from outside of a crystal.1 To design such a porous space, our group has intensively studied the metalloligand approach in which a pre-prepared homometallic complex with coordination donor sites is reacted stepwise with secondary metal ions. $2 \mathrm{We}$ established the construction of a variety of metalloarchitectures by the metalloligand approach with using thiolato groups derived from amino acids and phosphine ligands. Recently, our group has successfully prepared a microporous material of a nanometer-sized AuICdII 116-nuclear cage-of-cage structure (1CdNa) from the reaction of the tripodal-type trigold(I) metalloligand, [AuI3(tdme)(d-Hpen)3] (tdme = 1,1,1-tris(diphenylphosphinomethyl)ethane, d-H2pen = d-penicillamine), with CdII(NO3)2.3 The cage-of-cage structure was constructed from 12 building units of AuI6CdII3 cage complex through hierarchical aggregation. Interestingly, $1 \mathrm{CdNa}$ has large interstices connected by 3D channels which allow the easy incorporation and accommodation of guest molecules. Therefore, it was found that 1CdNa underwent the stepwise SCSC transmetallation reactions to form AuICuII metallocage $(1 \mathrm{Cu})$. Furthermore, we found that the crystals of $1 \mathrm{Cu}$ have the ability to accommodate MoO42- ions (2Mo1) and condense them to form Mo7O246- (2Mo7) and $\beta-M o 8 O 264-(2 \mathrm{Mo} 8)$ by the addition of protons in the solid state. These results show the availability of the large crystal interstices in $1 \mathrm{Cu}$ as crystal flask, which serves as a reaction field for accommodated chemical species in crystal. Such a crystal flask reaction of polyoxomolybdate will give an important insight for not only material science but also biosynthesis in Mo-storage protein (MoSto) which contains Mo8, Mo5-7 and Mo3 clusters. The detail will be discussed in the presentation.

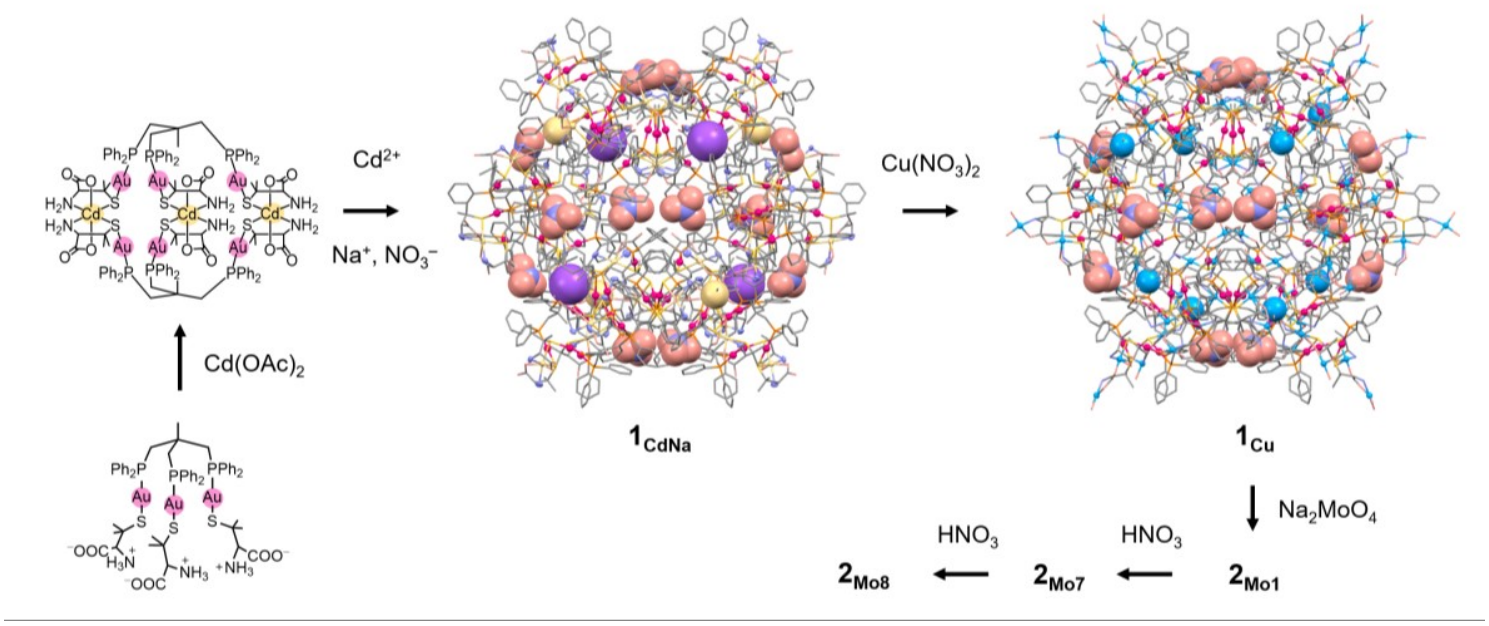

Figure 1. Synthetic route of $\mathbf{1}_{\mathrm{CdNa}}, \mathbf{1}_{\mathrm{Cu}}, \mathbf{2}_{\mathrm{Mo1}}, \mathbf{2}_{\mathrm{Mo}}$, and $\mathbf{2}_{\mathrm{Mos}}$.

[1] Inokuma, Y., Kawano, M. \& Fujita, M. (2011). Nat. Chem. 3, 349.

[2] Yoshinari, N. \& Konno, T. (2016). Chem. Rec. 16, 1647.

[3] Imanishi, K., Wahyudianto, B., Kojima, T., Yoshinari, N. \& Konno, T. (2020). Chem. Eur. J. $26,1827$.

[4] Kowalewski, B., Poppe, J., Demmer, U., Warkentin, E., Dierks, T., Ermler, U. \& Schneider, K. (2012). J. Am. Chem. Soc. 134, 9768.

\section{Keywords: Metallosupramolecules; Polyoxometalate Crystal Flask}

This research was Supported by CREST, JST (Grant No. JPMJCR13L3) and EPSRC-JSPS core-to-core program. 